\title{
Solution-processed ambipolar organic field-effect transistors and inverters
}

E. J. Meijer, D. M. de Leeuw, S. Setayesh, E. van Veenendaal, B.-H. Huisman, P.W. M. Blom, J. C. Hummelen, U. Scherf and T. M. Klapwijk

Nature Materials 2, 678-682 (2003).

J. Kadam was originally omitted from the author list of the above published paper. His name should have been listed after U. Scherf.

J. Kadam's affliation is Bergische Universitat Wuppertal, Department of Chemistry, Gauss-strasse 20, D-42097 Wuppertal, Germany. 